\title{
Efetividade de exercícios de estabilização segmentar sobre a dor lombar crônica mecânico-postural
}

\author{
Effectiveness of segmental stabilization exercises on mechanical-postural \\ chronic low back pain
}

\author{
Natália Toledo Pereira ${ }^{[a]}$, Luiz Alfredo Braun Ferreira ${ }^{[b]}$, Wagner Menna Pereira ${ }^{[c]}$
}

[a] Discente em Fisioterapia pela Universidade Estadual do Centro-Oeste (UNICENTRO), Itá, SC - Brasil, e-mail: nati_toledo@yahoo.com.br

[b] Mestre em Bioengenharia pela Universidade do Vale do Paraíba (UNIVAP), docente da Universidade Estadual do Centro-Oeste (UNICENTRO), Ponta Grossa, PR - Brasil, e-mail: luiz_braun@hotmail.com

[c] Mestre em Bioengenharia pela Universidade do Vale do Paraíba (UNIVAP), docente da Universidade Estadual do Centro-Oeste (UNICENTRO), Cascavel, PR - Brasil, e-mail: wagner.fisio@hotmail.com

\section{Resumo}

Introdução: A prevalência ao longo da vida de lombalgias mecânico-posturais é estimada em 60-70\% em países industrializados. Um dos principais fatores para dor lombar é a instabilidade segmentar, e para manter a estabilidade é necessária a interação de três subsistemas: passivo, ativo e controle neural. Exercícios específicos que promovem a contração independente dos músculos profundos do tronco (transverso do abdômen e multífido) têm demonstrado ter efeitos benéficos em indivíduos que sofrem de dor lombar inespecífica, sugerindo a estimulação desses subsistemas. Objetivo: Avaliar a efetividade de exercícios de estabilização segmentar sobre a dor e a capacidade funcional em indivíduos com lombalgia crônica. Materiais e métodos: Participaram da pesquisa 12 mulheres jovens com idade média de 20,66 \pm 3,74 anos. Foram realizadas 12 sessões de um programa de estabilização segmentar com frequência de duas vezes semanais, sendo avaliadas quanto à dor (questionário McGILL-Br) e capacidade funcional (questionário Rolland-Morris Brasil) antes e depois do período de intervenção. Resultados: Houve melhora significativa dos valores médios do índice de dor $(\mathrm{p}<0,0001)$, melhora do índice de dor sensitiva $(\mathrm{p}=0,0024)$, afetiva $(p=0,048)$, avaliativo $(p=0,042)$ e miscelânea $(p=0,017)$ e melhora da capacidade funcional dos indivíduos $(\mathrm{p}<0,0001)$, após o período de intervenção. Discussão: Vários estudos relataram a eficácia de exercícios dos músculos profundos do tronco, apresentando efeitos benéficos em indivíduos com lombalgia, corroborando com o estudo proposto. Conclusão: Pode-se concluir que o programa de 
estabilização segmentar foi efetivo na redução da dor e na melhora da função nestes pacientes, demonstrando assim ser um método eficaz de tratamento de lombalgias.

Palavras-chave: Dor Lombar. Terapia por exercício. Estabilização. Fisioterapia.

\begin{abstract}
Introduction: The lifetime prevalence of mechanical-postural low back pain is estimated at $60-70 \%$ in industrialized countries. One of the main factors for back pain is the segmental instability, and to keep the stability is required the interaction of three subsystems: passive, active and neural control. Specific exercises that promote independent contraction of deep muscles of the trunk (transverse abdominus and multifidus) have proven to have beneficial effects in individuals suffering from nonspecific low back pain, suggesting the stimulation of these subsystems. Objective: To evaluate the effectiveness of segmental stabilization, exercises on pain and functional ability in people with chronic LBP. Materials and methods: Surveyed 12 young women with an average age of $20.66 \pm 3.74$ years. Twelve sessions were held for a program frequently segmental stabilization of two times per week, being evaluated on pain (questionnaire McGILL-Br) and functional capacity (Roland-Morris). Results: There was a significant improvement of the mean values of pain index $(p<0.0001)$, improvement in sensory pain index $(p=0.0024)$, emotional $(p=0.048)$, evaluative $(p=0.042)$ and miscellaneous $(p=0.017)$ and functional capacity of the individuals $(p<0,0001)$, after the intervention period. Discussion: Many studies have reported the effectiveness of exercises of deep muscles of the trunk, showing beneficial effects in individuals with low back pain, corroborating with the proposed study. Conclusion: It can be concluded that the segmental stabilization program was effective in reducing pain and improving function in these patients, thus demonstrating to be an effective method of treatment of back pain.
\end{abstract}

Keywords: Low back Pain. Exercise therapy. Stabilization. Physical therapy.

\title{
Introdução
}

A prevalência ao longo da vida de lombalgias não específicas ou mecânico-postural (mais comum) é estimada em 60-70\% em países industrializados (1), sendo uma das causas mais frequentes de atendimento médico e a segunda causa de afastamento do trabalho (2). Episódios de lombalgia aguda (LBP) resolvem-se em apenas duas a quatro semanas para 90\% dos pacientes. No entanto, após o primeiro episódio da LBP, a recorrência da dor lombar ocorre em $60-80 \%$ dos pacientes $(3,4)$.

A dor lombar pode ser caracterizada por um quadro de desconforto e fadiga muscular localizada na região inferior da coluna vertebral (5). Dor lombar inespecífica é muitas vezes associada a lesões musculoesqueléticas e aos desequilíbrios na coluna lombar e estabilização dos músculos pélvicos (6).

A instabilidade segmentar ocorre quando há diminuição na capacidade do sistema estabilizador da coluna vertebral em manter a zona neutra dentro de limites fisiológicos (7). A zona neutra é uma região de movimentos intervertebrais onde pouca resistência é oferecida pela coluna vertebral passiva; a perda de controle da zona neutra no segmento vertebral está associada à lesão, doença degenerativa do disco e fraqueza muscular (8).

De acordo com Panjabi (9), a estabilidade da coluna consiste na interação de três subsistemas: passivo (articulações, ligamentos e vértebras), ativo (músculos e tendões) e controle neural (nervos e SNC). As funções desses três subsistemas estão interligadas, e a reduzida função de um subsistema pode colocar exigências crescentes sobre os outros.

Bermak (10) afirma que há dois sistemas musculares atuando sobre a estabilidade espinhal: o sistema global, que inclui o reto abdominal, oblíquo abdominal externo e a parte torácica lombar do iliocostal e proporciona a estabilização geral do tronco; e o sistema local, que é composto pelo multífido lombar, transverso 
abdominal, diafragma, fibras posteriores do oblíquo interno e quadrado lombar, responsáveis por fornecer estabilidade segmentar e controlar diretamente os segmentos lombares (11).

Graças à orientação das fibras horizontais, a contração do transverso abdominal resulta em redução da circunferência abdominal com um aumento da tensão na fáscia tóraco-lombar e da pressão intra-abdominal (PIA) (12). Este aumento da PIA faz com que o abdômen se transforme em um cilindro rígido $(13,14)$.

Hides et al. (15) demonstrou, por meio da ressonância magnética, que a contração do transverso do abdômen melhora a estabilização da região lombo-pélvica.

A contração do transverso do abdômen ocorre antes de um movimento e independe da direção do movimento realizado (16-19). Porém, o início da contração do transverso abdominal (TRAbd) está significativamente retardada em pacientes com dor lombar, sendo necessária maior perturbação para iniciar sua ativação (20-23), resultando em um déficit de controle motor e estabilização muscular ineficiente da coluna vertebral (21).

Segundo Richardson et al. (24), a independente contração do transverso abdominal diminui a frouxidão da articulação sacroilíaca em maior grau do que o padrão geral de exercícios abdominal, confirmando que o uso de contrações independente deste músculo é útil para a dor lombar.

As fibras laminares profundas do músculo multífido lombar são fundamentais para proteção articular e estabilidade, sendo que sua atrofia resulta em perda da estabilidade dinâmica da coluna lombar (25). De acordo com um estudo biomecânico in vitro, o multífido lombar contribui com 2/3 para o aumento da rigidez da coluna lombar (26).

Após um primeiro episódio de lombalgia unilateral, foi demonstrada uma redução rápida e segmentar do volume do músculo ipsilateralmente ao local de dor por meio de ultrassom (27). Kader et al. em seu estudo confirma que a atrofia do músculo multífido esteve presente em $80 \%$ dos pacientes com dor lombar (28).

Hides et al. observou também que a atrofia do multífido permanece mesmo após a resolução da dor lombar, aumentando assim as chances de recorrência de lombalgias (29).

Evidências encontradas em revisões sistemáticas sugerem que exercícios de estabilização segmentar que promovem cocontracão dos músculos transverso do abdômen e multífido são eficazes para reduzir a dor e a incapacidade em lombalgias crônicas e para aumentar o retorno às atividades diárias normais e ao trabalho $(30,31)$. Exercícios de estabilização parecem também ser mais eficazes do que o exercício de fortalecimento tradicional em dores lombares crônicas (32).

O’Sullivan, Twomey e Allison (33) investigaram o efeito do exercício de estabilização segmentar, comparando-a com exercício geral. A dor e incapacidade funcional foram significativamente reduzidas no grupo de exercício de estabilização segmentar após dez semanas de programa. Além disso, o efeito foi mantido nos 30 meses seguintes da avaliação.

Em um estudo realizado por Hides et al. (34) em indivíduos com episódio de lombalgia unilateral com atrofia do multífido foram realizados exércitos de treinamento específico para este músculo em cocontração com o transverso do abdômen, sendo observada diminuição da atrofia do multífido após o período intervenção, e em longo prazo (um ano após intervenção) a recorrência de dor lombar foi de apenas de 30\% no grupo de exercícios específicos - em contraste com $84 \%$ dos pacientes do grupo controle. Assim, os exercícios de estabilização segmentar mostraram mais uma vez ser mais eficazes na redução recorrência da dor lombar do que somente o tratamento médico.

Desta forma, o presente estudo tem por objetivo analisar a efetividade de exercícios de estabilização segmentar, sobre a dor em indivíduos com lombalgia inespecífica, contribuindo assim futuramente para a área de reabilitação de pacientes com dor lombar.

\section{Materiais e métodos}

\section{Amostra}

Para obtenção da amostra foram selecionados estudantes do curso de Fisioterapia da Universidade Estadual do Centro-Oeste (Unicentro). 
A amostra do trabalho foi constituída de 12 adultas jovens (18-32 anos) com idade média de 20,66 \pm 3,74 anos que apresentaram dor lombar crônica inespecífica. Foram excluídos sujeitos com característica de diagnóstico bem definido como dor ciática, frouxidão da articulação sacroilíaca, espondilólise, doença neurodegenerativa, infecciosa ou inflamatória.

O estudo teve a aprovação do Comitê de Ética em Pesquisa em Seres Humanos da Unicentro, protocolo 166/2009, com base na Resolução 196/96 do Conselho Nacional de Saúde.

\section{Instrumentos e procedimentos}

Para avaliação mais detalhada da dor e capacidade funcional dos indivíduos foram utilizados questionários pré e pós-tratamento.

Foi aplicado o Questionário de Roland-Morris para avaliar a interferência da lombalgia na função do paciente, que consiste de 24 itens que descrevem situações de vida diária que podem ser influenciadas por dores lombares. Escores mais altos indicam maior incapacidade (35).

O questionário de dor McGILL-Br MPQ (versão brasileira) foi utilizado para avaliar a características da dor, além da sua intensidade. Ele é constituído por quatro grupos (sensitivo-descriminativo, afetivo-motivacional, cognitivo-avaliativo e miscelânea), 20 subgrupos e 78 descritores. A partir do questionário pode-se chegar às seguintes medidas: número de descritores escolhidos e índice de dor. Além disso, o questionário utiliza uma escala verbal que utiliza cinco palavras graduadas de 0 a 5 pontos para caracterizar a intensidade da dor naquele momento (36).

O tratamento de estabilização segmentar foi aplicado em duas sessões semanais com duração aproximadamente de 35 minutos durante o período de seis semanas, sendo realizado em um grupo de 12 pacientes, com o terapeuta dando estímulos auditivos e táteis para a realização correta dos exercícios.

Foi realizado um protocolo de tratamento que consiste em seis etapas progressivas de exercícios, realizando a contração isométrica do transverso do abdômen e multífido, assim como a contração da musculatura do assoalho pélvico seguindo as seguintes orientações do terapeuta: "puxe o ar - solte - prenda a respiração"; "vagarosamente puxe sua barriga para dentro e segure"; "puxe para cima e para dentro seu esfíncter".

Cada exercício foi realizado em 12 repetições mantendo a contração durante 10 segundos. Os pacientes foram também orientados a realizar os exercícios diariamente em suas residências.

\section{Etapa 1 (1 ${ }^{\circ}$ semana): sem carga, estático, sem equilíbrio/perturbação}

Em supino, com os joelhos flexionados em adução, ativação do músculo transverso do abdômen.

Em prono, joelhos estendidos e braços ao longo do corpo, ativação do músculo multífido.

\section{Etapa 2 ( $2^{\circ}$ semana): sem carga, com movimento, sem equilíbrio/perturbação}

Em supino, joelhos flexionados, movimentos com o membro inferior (deslizamento do calcanhar).

Em supino, movimentos alternados dos membros, elevação unilateral do membro superior e flexão do membro inferior contralateral (dead bug).

Etapa 3 ( $3^{\circ}$ semana): sem carga, com movimento, com equilíbrio/perturbação

Ponte, com os pés apoiados no solo e joelhos flexionados, elevar a pelve mantendo a contração da musculatura profunda do tronco.

Posição de gatos com movimentos alternados em diagonal dos membros superiores (flexão) e inferior (extensão).

Etapa 4 ( $4^{\circ}$ semana): com carga, sem movimento, sem equilíbrio/perturbação

Sentado, realiza rolamento pélvico (empinar os glúteos sem empinar o tórax) e contração da musculatura profunda do tronco.

Em pé estático, realiza a contração do transverso do abdômen e multífido. 
Etapa 5 ( $5^{\circ}$ e $6^{\circ}$ semana): core training - exercícios mais intensos ativando toda a musculatura que envolve a coluna vertebral, promovendo assim maior estabilidade

Ponte unilateral: elevação da pelve associada à elevação de um membro inferior que é mantido em extensão, mantendo a contração do transverso do abdômen e multífido.

Ponte lateral: em decúbito lateral, realiza elevação lateral da pelve com apoio nos pés e cotovelo, mantendo a contração do transverso do abdômen e multífido.

Prancha: em decúbito ventral, realiza elevação da pelve com apoio nos pés e cotovelo, mantendo a contração do transverso do abdômen e multífido.

\section{Análise estatística}

A análise estatística foi realizada de forma descritiva e inferencial. O teste de D Agostino foi utilizado para testar a normalidade ou não da amostra, com significância de $95 \%(\mathrm{p}<0,05)$. Utilizou-se o teste de Wilcoxon não paramétrico, com nível de significância de $95 \%(\mathrm{p}<0,05)$ para comparação das medias pré e pós-tratamento e o teste " $t$ " de Student para amostras paramétricas.

\section{Resultados}

Foi possível observar que houve melhora significativa da dor e capacidade funcional dos indivíduos, após o período de intervenção.

As características e índice da dor foram avaliados por meio do questionário McGILL-Br (versão brasileira), constituído por quatro grupos: grupo das respostas sensitivas à experiência dolorosa, como propriedades mecânicas e térmicas; grupo de dimensões afetivas, que se refere à tensão e medo; grupo de descritores avaliativos da experiência dolorosa global; e miscelânea (36). Houve uma redução na média do índice de dor em todos os grupos citados, com diferenças significativas de $\mathrm{p}<0,05$ : sensitiva $(\mathrm{p}=0,0024)$, afetiva $(\mathrm{p}=$ $0,048)$, avaliativo $(p=0,042)$ e miscelânea $(p=0,017)$ (Gráfico 1).

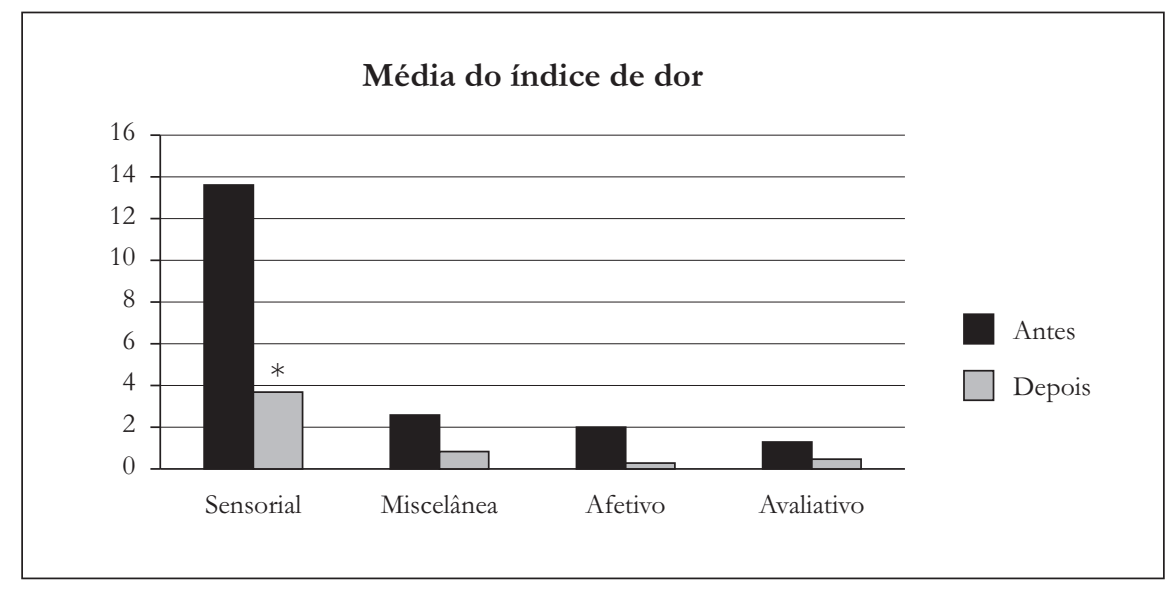

Gráfico 1 - Comparação da média do índice de dor nos grupos antes e depois do período de intervenção, por meio do questionário McGILL-Br Nota: $*$ Diferença significativa nos valores de média $(\mathrm{p}<0,05)$.

O questionário McGILL-Br (versão brasileira) utiliza ainda uma escala verbal constituída de cinco palavras graduadas de 0 a 5 pontos, em que sem dor $=0$, fraca $=1$, moderada $=2$, forte $=3$, violenta $=4$, 
insuportável $=5$ pontos, para caracterizar a intensidade da dor naquele momento (36). As médias dos valores de dor reduziram de 2,25 $\pm 0,45$ inicial para $0,83 \pm 0,57$ após o período de intervenção, com diferença significativa de $\mathrm{p}<0,0001$ (Gráfico 2).

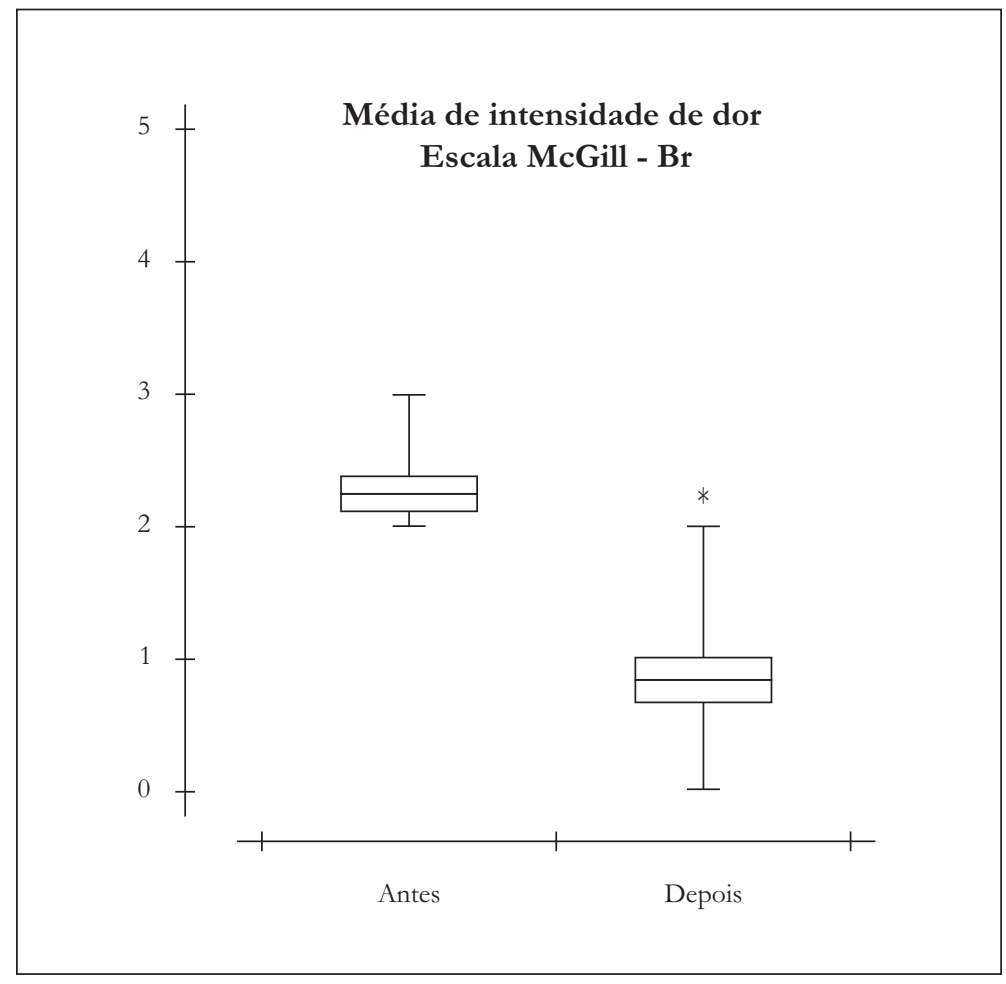

Gráfico 2 - Comparação da média da intensidade de dor antes e após o período de intervenção, por meio do questionário McGILL-Br

Nota: *Diferença significativa nos valores de média $(\mathrm{p}<0,0001)$.

Observou-se, por meio do questionário Roland-Morris Brasil, que houve melhora funcional com uma redução da média de 2,75 $\pm 1,13$ pontos inicial para 1,33 $\pm 0,65$ pontos após a intervenção, com diferença estatisticamente significativa de $\mathrm{p}<0,0001$ (Gráfico 3).

\section{Discussão}

No presente estudo avaliou-se a efetividade de exercícios de estabilização segmentar lombar na redução da dor e incapacidade em indivíduos com dor lombar inespecífica.

Os resultados demonstram que após seis semanas de exercícios de estabilização segmentar, que consistem na contração da musculatura profunda do tronco, transverso do abdômen e multífido, houve melhora da dor e capacidade funcional dos indivíduos.

Está melhora ocorreu apesar de os indivíduos não apresentarem na avaliação inicial um nível muito elevado de dor e indícios de incapacidade funcional, sendo observada diferença significativa pré e pós-intervenção.

Estes resultados estão de acordo com vários outros estudos nos quais foi demonstrado que exercícios de estabilização segmentar, comparados a nenhuma intervenção ou a atendimento médico geral, têm sido mais eficazes para a redução da dor lombar a curto e em longo prazo (37-39). 


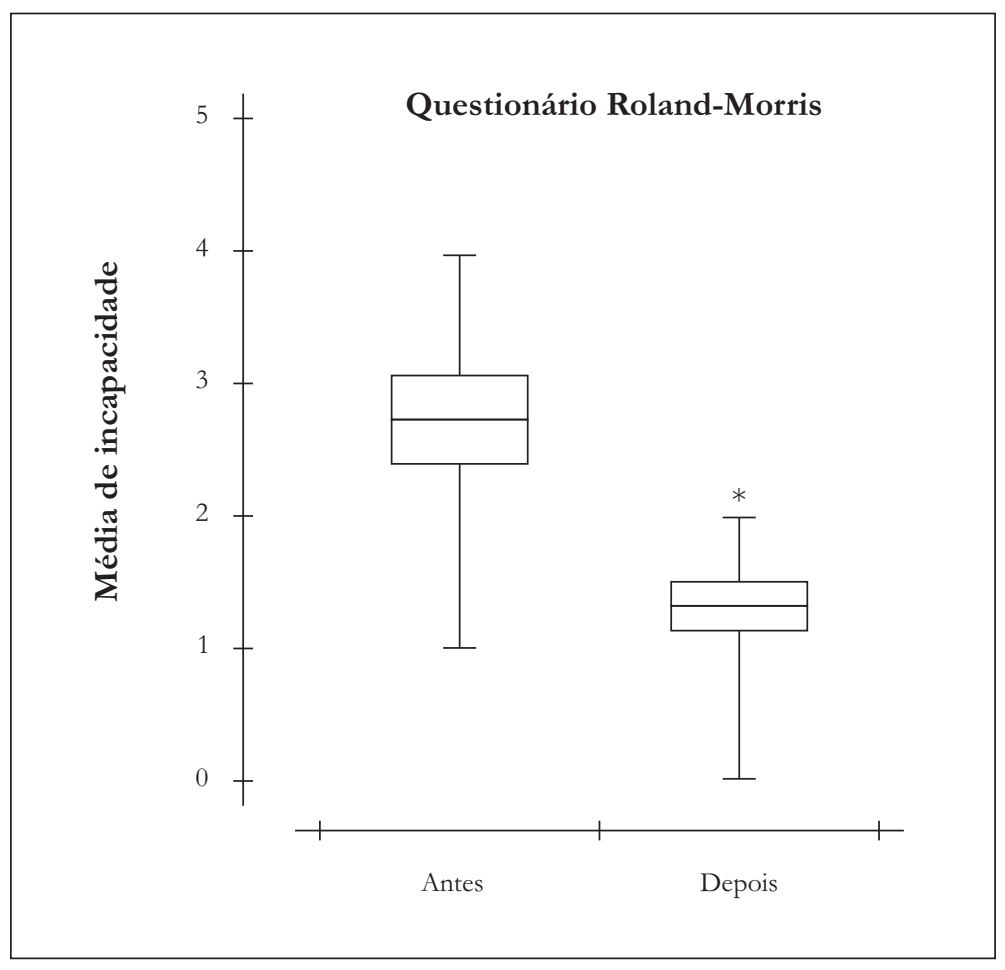

Gráfico 3 - Comparação entre a média de pontuação antes e depois do período de intervenção, por meio do questionário Roland-Morris

Nota: $*$ Diferença significativa nos valores de média $(\mathrm{p}<0,0001)$.

Um estudo comparou o tratamento de fisioterapia convencional (ultrassom e diatermia de ondas curtas e exercícios de fortalecimento lombar) a técnicas de estabilização segmentar em jogadores de hóquei jovens com dor lombar crônica e subaguda e foi demonstrado que a estabilização segmentar pareceu ser mais eficaz que o tratamento convencional (40).

Leite et al. (41) relatou uma melhora uma melhora da dor e desempenho funcional após o tratamento de 12 sessões de um programa de exercícios de estabilização segmentar em pacientes com dor lombar crônica inespecífica. Estes resultados mostram, como no presente estudo, que é possível reduzir a dor lombar em pacientes com um quadro de lombalgia crônica em um período relativamente curto de intervenção.

Os achados de Ferreira et al. (42) demonstram a eficácia de exercícios específicos para o transverso do abdômen, em comparação a exercícios gerais e terapia manipulativa espinhal em pacientes com dor lombar crônica. O efeito do exercício de controle motor na redução da dor foi maior em comparação com os outros grupos e houve ainda uma correlação significativa entre o recrutamento moderado do transverso abdominal e diminuição da incapacidade, demonstrando assim mais uma vez a importância da ação deste músculo na estabilidade da coluna lombar.

Em outro estudo que também realizou um programa de exercícios de estabilização segmentar durante seis semanas em indivíduos com dor lombar crônica, foi possível observar diferenças significativas entre os grupos de intervenção e controle no Questionário de Rolland-Morris e Short Form McGill Pain Questionnaire (SF-MPQ) ( $<$ ou = 0,0071). Sendo que 89\% dos pacientes consideraram o seu nível de dor e incapacidade funcional aceitável após o programa de estabilização segmentar, que foi constituído por três etapas progressivas, demonstrando assim, igualmente ao nosso estudo, que houve redução significativa da dor e incapacidade no grupo de sujeitos estudados (43). 
Godly et al. (44) demonstrou ainda a eficácia de exercícios de controle motor em comparação com a terapia manual. No grupo de estabilização segmentar, a dor e incapacidade funcional melhoraram significativamente após o período de tratamento e se mantiveram em longo prazo.

Portanto, exercícios específicos que promovem a contração independente dos músculos profundos do tronco, cocontracão do transverso do abdômen e multífido têm sido demonstrado ter efeitos benéficos na redução da dor e incapacidade em pacientes com lombalgia crônica e na diminuição de taxas de recorrência após um episódio de dor aguda $(24,33,34)$.

\section{Conclusão}

Os resultados obtidos neste estudo demonstram que um programa de seis semanas de exercícios de estabilização segmentar com frequência de duas vezes semanais foi efetivo na redução da dor e na incapacidade funcional na dor lombar mecânico-postural em mulheres, demonstrando assim ser um método eficaz de tratamento de lombalgias. Entretanto, mais estudos são necessários para avaliar os efeitos de exercícios de estabilização em pacientes com lombalgia, utilizando amostra maior de pacientes e comparações com um grupo controle.

\section{Referências}

1. Burton AK, Balagué F, Cardon G, Eriksen HR, Henrotin Y, Lahad A, et al. How to prevent low back pain. Best Pract Res Clin Rheumatol. 2005;19(4):541-55.

2. Ebenbichler GR, Oddsson LIE, Kollmitzer J, Erim Z. Sensory-motor control of the lower back: implications for rehabilitation. Med Sci Sports Exerc. 2001;33(11):1889-98.

3. Hides JA, Richardson CA, Jull GA. Multifidus muscle recovery is not automatic after resolution of acute, firstepisode low back pain. Spine. 1996;21(23):2763-9.

4. Hides JA, Richardson CA, Jull GA. Magnetic resonance imaging and ultrasonography of the lumbar multifidus Muscle. Spine. 1995;20(1):54-8.

5. Reineh FB, Carpes FP, Mota CB. Influência do treinamento de estabilização central sobre a dor e estabilidade lombar. Fisioter Mov. 2008;21(1):123-9.

6. Vogt L, Pfeifer K, Banzer W.Neuromuscular control of walking with chronic low-back pain. Man Ther. 2003;8(1):21-8.

7. Fritz JM, Erhard RE, Hagen BF. Segmental instability of the lumbar spine. Phys Ther. 1998;78(8):889-96.

8. Panjabi, M. The stabilizing system of the spine. Part II. Neutral zone and instability hypothesis. J Spinal Disord. 1992;5(4):390-6.

9. Panjabi, M. The stabilizing system of the spine. Part I. Function, dysfunction, adaptation, and enhancement. J Spinal Disord. 1992;5(4):383-9.

10. Bergmark A. Stability of lumbar spine: a study in mechanical engineering. Acta Orthop Scand. 1989;230 (Suppl 1):1-54.

11. O'Sullivan PB. Lumbar segmental "instability": clinical presentation and specific stabilizing exercise management. Man Ther. 200;5(1):2-12.

12. Hodges PW. Is there a role for transverses abdominis in lumbo-pelvic stability? Man Ther. 1999;4(2):74-86.

13. McGill SM, Cholewicki J. Biomechanical basis for stability: an explanation to enhance clinical utility. J Orthop sport Phys Ther. 2001;31(2):96-100. 
14. Hodges PW, Cresswell AG, Daggfelddt K, Thorstensson A. In vivo measurement of the effect of intra-abdominal pressure on the human spine. J Biomech. 2001;34(3):347-53.

15. Hides J, Wilson S, Stanton W, McMahon S, Keto H, McMahon K, et al. An MRI investigation into the function of the transversus abdominis muscle during "drawing-in" of the abdominal wall. Spine. 2006;31(6):175-8.

16. Hodges PW, Richardson CA. Feedforward contraction of transversus abdominis is not influenced by the direction of arm movement. Exp Brain Res. 1997;114(2):362-70.

17. Hodges PW, Richardson CA, Hasan Z. Contraction of the Abdominal Muscles Associated With Movement of the Lower Limb. Phys Ther. 1997;77(2):132-44.

18. Hodges P, Cresswell A, Thorstensson A. Preparatory trunk motion accompanies rapid upper limb movement. Exp Brain Res. 1999;124(1):69-79.

19. Hodges PW, Richardson CA. Transversus abdominis and the superficial abdominal muscles are controlled independently in a postural task. Neurosci Lett. 1999;265(2):91-4.

20. Hodges PW, Richardson CA. Altered trunk muscle recruitment in people with low back pain with upper limb movement at different speeds. Arch Phys Med Rehabil. 1999;80(9):1005-12.

21. Hodges PW, Richardson CA. Inefficient muscular stabilization of the lumbar spine associated with low back pain: a motor control evaluation of transversus abdominis. Spine. 1996;21(22):2640-50.

22. Hodges PW, Richardson CA. Delayed postural contraction of transversus abdominis in low back pain associated with movement of the lower limb. J Spinal Disord. 1998;11(1):46-56.

23. Hodges PW. Changes in motor planning of feedforward postural responses of the trunk muscles in low back pain. Exp Brain Res. 2001;141(2):261-6.

24. Richardson CA, Snijder CJ, Hides JA, Damen L, Pas MS, Stom J. The relationship between the transversus abdominis muscles, sacroiliac joint mechanics, and low back pain. Spine. 2002;27(4):399-405.

25. Watson T, McPherson S, Starr K. The association of nutritional status and gender with cross-sectional area of the multifidus muscle in establishing normative data. J Man Manip Ther. 2008;16(4):E93-8.

26. Wilke HJ, Wolf S, Claes LE, Arand M, Weisend A. Stability increase of the lumbar spine with different muscle groups: a biomechanical in vitro study. Spine. 1995;15;20(2):192-8.

27. Hides JA, Stokes MJ, Saide M, Jull GA, Cooper DH. Evidence of lumbar multifidus muscle wasting ipsilateral to symptoms in patients with acute/subacute low back pain. Spine. 1994;19(2):165-72.

28. Kader DF, Wardlaw D, Smith FW. Multifidus muscle atrophy sides in the MRI and leg pain side. Clinical Radiology. 2000;55:145-9.

29. Hides JA, Richardson CA, Jull GA. Multifidus muscle recovery is not automatic following resolution of acute first-episode low back pain. Spine. 1996; 21(23):2763-9.

30. Ferreira PH, Ferreira ML, Maher CG, Herbert RD, Refshauge K. Specific stabilisation exercise for spinal and pelvic pain: a systematic review. Aust J Physiother. 2006;52(2):79-88.

31. Van Tulder M, Malmivaara A, Esmail R, Koes B. Exercise therapy for low back pain: a systematic review within the framework of the cochrane collaboration back review group. Spine. 2000;25(21):2784-96.

32. Kasai R, RPT MA. Current trends in exercise management for chronic low back pain: comparison between strengthening exercise and spinal segmental stabilization exercise. J Phys Ther Sci. 2006;18(1):97-105.

33. O'Sullivan PB, Twomey LT, Allison GT. Evaluation of specific stabilization exercise in the treatment of chronic low back pain with radiologic diagnosis of spondylolysis or spondylolisthesis. Spine. 1997;22(24):2959-67. 
34. Hides JA, Jull GA, Richardson CA. Long-term effects of specific stabilizing exercises for first-episode low back pain. Spine. 2001;26(11):243-8.

35. Nusbaum L, Natour J, Ferraz MB, Goldenberg J. Translation, adaptation and validation of the Roland-Morris questionnaire - Brazil Rolan-Morris. Braz J Med Biol Res. 2001;34(2):203-10.

36. Pimenta CAM, Teixeira MJ. Questionário de dor McGill: proposta de adaptação para a língua portuguesa. Rev Esc Enf USP. 1996;30(3):473-83.

37. Moseley L. Combined physiotherapy and education is efficacious for chronic low back pain. Aust J Physiother. 2002;48(4):297-302.

38. Niemisto L, Lahtinen-Suopanki T, Rissanen P, Lindgren KA, Sarna S, Hurri H. A randomized trial of combined manipulation, stabilizing exercises, and physician consultation compared to physician consultation alone for chronic low back pain. Spine. 2003;28(19):2185-91.

39. Shaughnessy M, Caulfield B. A pilot study to investigate the effect of lumbar stabilisation exercise training on functional ability and quality of life in patients with chronic low back pain. Int J Rehabil Res. 2004;27(4):297-301.

40. Kumar S, Sharma VP, Negi MP. Efficacy of dynamic muscular stabilization techniques (DMST) over conventional techniques in rehabilitation of chronic low back pain. J Strength Cond Res. 2009;23(9):2651-9.

41. Leite MRR, Oliveira TH, Mello RM, Valadas M, Nascimento MR, Freitas AE, et al. Efetividade dos exercícios de estabilização lombo-pélvico no tratamento da dor lombar crônica não-específica. Terapia Manual. 2008,6(27):270-5.

42. Ferreira P, Ferreira M, Maher C, Refshauge K, Herbert R, Hodges P. Changes in recruitment of transversus abdominis correlate with disability in people with chronic low back pain. Br J Sports Med. 2010 May 10. [Epub ahead of print].

43. Norris C, Matthews M. The role of an integrated back stability program in patients with chronic low back pain. Complement Ther Clin Pract. 2008;14(4):255-63.

44. Goldby L, Moore A, Doust J, Trew M. A randomized controlled trial investigating the efficiency of musculoskeletal physiotherapy on chronic low back disorder. Spine. 2006;31(10):1083-93.

Recebido: 08/07/2010

Received: 07/08/2010

Aprovado: 03/09/2010

Approved: 09/03/2010 